\title{
Da Saúde Materno Infantil ao PAISM
}

Daphne RATTNER ${ }^{1}$

A criação do Programa de Atenção Integral à Saúde da Mulher (PAISM) em 1984 foi um marco político e histórico. Foi resultado das reivindicações dos movimentos de mulheres e movimentos feministas pelo direito ao atendimento à saúde integral, ou seja: ações de saúde dirigidas para o atendimento global das necessidades das mulheres. 1

Até então a visão que prevalecia era a da atenção materno-infantil, cujos princípios norteadores de política de saúde para as mulheres eram baseados em sua especificidade biológica e no seu papel social de mãe. No bojo de um movimento internacional de crescimento da consciência das mulheres de seu papel social historicamente construído, que estava sendo decodificado adotando o enfoque de gênero, e incorporando a possibilidade de planejar o número de filhos e a oportunidade de engravidar, assim como integrando a revinidicação pelo direito ao aborto legalizado, o movimento de mulheres brasileiro conseguiu que fossem separadas as duas áreas técnicas em (1) Saúde da Criança e do Adolescente e (2) Saúde da Mulher.

Essa ruptura conceitual foi radical e contribuiu para que a mulher fosse vista não apenas como quem engravida e tem filhos, mas também como sujeito de direitos, com a garantia de atendimento a outras questões de saúde além das relacionadas à reprodução social e fisiológica. Concomitantemente ocorria o processo de redemocratização, bem como a aprovação da Constituição Federal de 1988 que, entre outros avanços, criou o Sistema Único de Saúde com alicerce nos princípios da universalidade do acesso, integralidade e equidade nas ações de saúde e participação social e autonomia dos sujeitos.2

Constava no programa original do PAISM que as atividades básicas de atenção integral à saúde da mulher constituem um conjunto de ações educativas e preventivas, de diagnóstico, tratamento ou recuperação, aplicadas permanente e racionalmente, tendo como objetivo final a melhoria dos níveis de saúde da população feminina.1

Neste ano de 2013, quase trinta anos desde esse surgimento, embora o Brasil tenha mudado muito, incluindo a situação das mulheres, especialmente no campo dos direitos e do acesso à saúde, 1 Doutora em Epidemiologia pela University of North Carolina. É Professora Adjunta na Disciplina de Epidemiologia, no Departamento de Saúde Coletiva, Faculdade de Ciências da Saúde, na Universidade de Brasília. Contato: daphne. rattner@gmail.com 
ainda é difícil sua garantia no cotidiano, em particular daquelas que se encontram em situação de vulnerabilidade social, sobretudo no que diz respeito à classe, raça/etnia, geração, orientação sexual e identidade de gênero.

O PAISM constitui a base dos esforços governamentais relacionados ao campo das políticas estratégicas e programas do sistema de saúde na área da saúde da mulher. Em 2004, quando esse programa tomou a forma da Política Nacional de Atenção Integral à Saúde da Mulher, a definição das prioridades baseou- se em um diagnóstico da situação de saúde das mulheres no Brasil elaborado em conjunto com diversos atores sociais. 3,4

Esta política incorpora a integralidade, o enfoque de gênero, de raça e etnia e a promoção da saúde como princípios norteadores. Assim, preenche antigas lacunas ao introduzir as ações de atenção ao climatério, as queixas ginecológicas, a reprodução humana assistida, atenção ao abortamento inseguro e a segmentos da população feminina historicamente alijados das políticas públicas, nas suas especificidades e necessidades, tais como: mulheres em situação de prisão, mulheres negras, índias, lésbicas e bissexuais, trabalhadoras rurais, com deficiência. Também contempla o apoio à participação do movimento de mulheres no processo de elaboração, execução e avaliação da política de atenção integral à saúde da mulher, pelo reconhecimento de sua contribuição técnica e política no campo dos direitos e da saúde da mulher.

Atualmente, a Saúde da Mulher no Brasil atua em três grandes áreas prioritárias, além da atenção obstétrica: planejamento reprodutivo, câncer ginecológico e atenção a mulheres e adolescentes em situação ou risco de violência. 5

Com relação a essa atuação, nesse período houve grandes conquistas, principalmente no que se refere ao acesso aos serviços de saúde:

1) Em termos de planejamento reprodutivo, as mulheres conseguiram regular a sua fecundidade: se em 1960 cada mulher tinha em média 6,2 filhos, em 2006 a média foi de 1,8 filhos por mulher.6

2) Na atenção ao pré-natal: em 1995, para cada parto atendido no SUS, era realizada 1,2 consulta de pré-natal. Em 2011, foram quase 20 milhões de consultas de pré-natal para cerca de 2 milhões de partos atendidos no SUS, superando o preconizado para uma boa atenção ao pré-natal, ou seja, 6 consultas: foram 10,2 consultas para cada parto atendido, sendo mais de um terço dessas consultas realizadas por enfermeiras.

O aumento da cobertura de pré-natal, decorrente da ampliação da cobertura da Estratégia Saúde da Família, resultou em importante redução da mortalidade infantil, mostrando que o trabalho em equipe multiprofissional em um modelo de atendimento mais abrangente tem efeitos importantes na saúde das pessoas.

3) Aumentou o acesso ao exame preventivo do câncer de colo do útero, principalmente para // Tempus, actas de saúde colet, Brasília, 8(2), 103-108, jun, 2014.

ISSN 1982-8829 
mulheres a partir dos 25 anos de idade, portanto detecção precoce do câncer de mama está mais acessível, através da possibilidade de realização da mamografia a cada dois anos, principalmenbte a partir de 50 anos de idade.

4) Construção de redes estaduais e municipais de Atenção Integral à Saúde de Mulheres e Adolescentes em Situação de Violência Doméstica e Sexual.

Além desses grandes campos de atuação, também atendendo às reivindicações dos movimentos de mulheres, a ATSM tem procurado elaborar projetos específicos para determinados grupos de população feminina, cada qual com as suas especificidades, como as Mulheres negras, Mulheres do campo, da floresta e da cidade, Lésbicas e Mulheres que fazem sexo com Mulheres, Mulheres que vivenciam a transexualidade e transexuais, Mulheres com deficiência, Mulheres em situação de prisão, Mulheres vivendo com HIV/ AIDS, Mulheres quilombolas, Mulheres ciganas, Mulheres prostitutas e Mulheres vivendo em situação de rua. A Área Técnica de Saúde da Mulher também elaborou um manual para orientar profissionais no atendimento a mulheres no climatério/ menopausa. 3,7

Ainda que se contabilizem avanços, os desafios atuais não são poucos:

1) Apesar de ter aumentado a distribuição de métodos contraceptivos reversíveis e ampliado o acesso à laqueadura e vasectomia e criando a possibilidade da mulher adquirir anticoncepcionais a preços módicos na Farmácia Popular, em muitas regiões o suprimento ainda não é regular e nem todos os métodos estão acessíveis. E ainda são poucos os serviços de reprodução humana assistida disponibilizados às pessoas que pretendem engravidar e que não conseguem.

2) Embora tenha sido ampliado o acesso ao pré-natal, há problemas sérios em relação à sua qualidade, o que é revelado pelos altos números de casos de sífilis congênita, 8 a continuidade de casos de tétano neonatal e, principalmente, pela alta razão de mortalidade materna9- sendo que análises mostram que mais de $90 \%$ dos óbitos maternos seriam evitáveis de alguma forma.

3) Há também problemas sérios no atendimento humanizado ao abortamento inseguro e há dificuldades para a realização do aborto previsto em lei.

4) Além disso, também a qualidade da assistência ao parto é problemática, visto que o Brasil ostenta um dos mais altos índices de cirurgia cesariana no parto (dados de 2011 revelaram que se encontra em 55\% no país, mais de $40 \%$ no SUS e mais de $80 \%$ na Saúde Suplementar) sugerindo que muitas delas não seriam necessárias. E, embora atendam mulheres em locais que o sistema de saúde é limitado, as parteiras tradicionais ainda não são reconhecidas pelo sistema de saúde.

5) O câncer de mama é a primeira causa de morte de mulheres por câncer e ainda há desigualdades regionais: a região Norte do país é a única em que o câncer de colo de útero é a principal causa de morte de mulheres por câncer, mostrando que um método simples de detecção precoce, ou 
seja, o exame citopatológico ou de Papanicolaou, ainda não está acessível nessa região. Aumentou também o câncer de pulmão em mulheres, diretamente associado ao aumento do hábito de fumar nessa população.

6) Há ainda outros problemas que vêm sendo abordados pelo Ministério da Saúde, mas que estão relacionados ao estilo de vida adotado pelas mulheres, como o aumento de doenças cardiovasculares em mulheres. Outras estão mais relacionadas às questões de gênero e de vulnerabilidades das mulheres como é o caso da feminização da epidemia de Aids e outras DST, a gravidez na adolescência e outros.

\section{A REDE CEGONHA}

A política proposta pela Presidência da República alcunhada de "Rede Cegonha" vem ao encontro da perspectiva de qualificação da atenção, reconhecendo como problemas prioritários a serem enfrentados os da mortalidade materna e na infância (até os cinco anos de idade): não morrer de uma morte evitável é uma questão ética e de Direitos Humanos, e configura-se como o grande desafio contemporâneo a ser enfrentado.

A Rede Cegonha aloca importante parcela dos recursos na priorização da atenção ao período reprodutivo, visando qualificar a atenção ao planejamento reprodutivo, à gestação, a partos e nascimentos, ao pós-parto e aos primeiros dois anos de vida, privilegiando a saúde das mulheres e o atendimento integral à criança a partir de seu nascimento. Nela são incorporadas: a passagem da compreensão biomédica, com foco no corpo da mulher e da criança, para a abordagem humanizada, interdisciplinar, com foco na família; as concepções mais atualizadas das práticas de atenção, fundamentadas na Medicina Baseada em Evidências e numa abordagem humanizada que consideraas mulheres como sujeitos partícipes da atenção.

Os avanços embutidos na proposta da Rede Cegonha dizem respeito por um lado à gestão do cuidado, incorporando os dispositivos da Política Nacional de Humanização, como o acolhimento com classificação de risco; o projeto terapêutico singular, traduzido como plano de expectativas para o parto e a ser implantado onde seja possível; o trabalho em equipe multiprofissional de forma transdisciplinar e complementar; aprimoramento do processo de trabalho, com adoção de atenção humanizada e práticas com base em evidências científicas; e outros.

Porém, além da gestão do cuidado, a proposta visa estruturar a gestão do sistema, com a organização da rede de atenção à saúde perinatal em níveis de assistência regionalizados e hieraquizados, com garantia de vaga a partir do início do pré-natal, diversidade de pontos de atenção, efetividade da regulação e ordenamento do sistema.11

Portanto, a Rede Cegonha representa o avanço no sentido de proteção integral ao processo de gestação- parto-nascimento-puerpério e início da vida tanto em termos da gestão do cuidado, como da gestão do sistema, fazendo parte de uma política nacional ampliada de atenção integral à saúde // Tempus, actas de saúde colet, Brasília, 8(2), 103-108, jun, 2014. 
das mulheres e das crianças. Como uma das principais inovações, saliente-se a diversificação de pontos de atenção, como Centros de Parto Normal; Casa de gestante de alto risco; alojamento para mães de bebês que ficaram internados; e outros.

Finalizando, nesse período desde a instituição do PAISM o Brasil mudou muito. Mas ainda é difícil o cotidiano das mulheres, principalmente aquelas que vivem numa situação de vulnerabilidade de classe social e racial/étnica, ou seja, as pobres, as negras e indígenas. As mulheres são as principais usuárias do SUS, vivem mais que os homens, mas adoecem com mais freqüência. E, para além do campo da saúde, o PAISM pode ser considerado um "produto" ou um "resultado" da mobilização das mulheres brasileiras pelo reconhecimento de cidadania e de acesso aos postos de decisão no espaço público.

\section{REFERÊNCIAS BIBLIOGRÁFICAS}

1 - BRASIL/Ministério da Saúde. Assistência Integral à Saúde da Mulher: bases de ação programática. Brasília: Ministério da Saúde, 1984.

2 - VIOLA RC. Políticas de atenção à saúde da mulher e os 20 anos de Sistema Único de Saúde no Brasil. In: Saúde Brasil 2008. 20 anos de Sistema Único de Saúde (SUS) no Brasil. Brasília: Ministério da Saúde, 2009.

3 - BRASIL/Ministério da Saúde. Política Nacional de Atenção Integral à Saúde da Mulher: Princípios e Diretrizes. Brasília: Ministério da Saúde, 2004a

4 - BRASIL/Ministério da Saúde. Política Nacional de Atenção Integral à Saúde da Mulher: Plano de Ação: 2004-2007. Brasília: Ministério da Saúde, 2004b

5 - RATTNER D. Saúde da Mulher. In: Ministério da Agricultura, Pecuária e Abastecimento (MAPA). Cooperativismo de Gênero. Brasília: MAPA, 2009.

6 - BRASIL/Ministério da Saúde, Cebrap. Pesquisa Nacional sobre Demografia e Saúde 1996 - PNDS 2006, Brasília: Ministério da Saúde, 2008

7 - BRASIL/Ministério da Saúde. Relatório de gestão 2003-2006: Política Nacional de Atenção Integral à Saúde da Mulher. Brasília: Ministério da Saúde, 2007

8 - BRASIL/Ministério da Saúde. Boletim Epidemiológico Sífilis 2012. Ano I, nº 1. Brasília: Ministério da Saúde, 2012a.

9 - BRASIL/Ministério da Saúde. Mortalidade materna no Brasil. Boletim 1/2012. disponível em http://portalsaude.saude.gov.br/portalsaude/index. $\quad$ cfm?portal=pagina. visualizarTexto\&codConteudo- $\quad=6403 \&$ codModuloArea=783\&chamada=boletim1/2012-_-mortalidade-materna-no-\%20\%20brasil. 
$108 / /$

Acesso em 09/01/2013. 2012b.

10 - BRASIL/Ministério da Saúde. Rede Cegonha. Diretrizes Gerais e Operacionais da Rede Cegonha. Disponível em http://portal.saude.gov.br/portal/ saude/gestor/visualizar_texto. cfm?idtxt=37082. Acesso em 20/01/2013.

11 - RATTNER D. Panorama da Rede Cegonha à luz dos direitos humanos. Versão preliminar. Documento solicitado pela Área Técnica de Saúde da Mulher - Ministério da Saúde. Mimeo, 2011.

Artigo apresentado em 11/12/13 Artigo aprovado em 21/01/14 Artigo publicado no sistema: 21/03/14 